\title{
Pesticide exposure of honeybees (Apis mellifera) pollinating melon crops
}

\author{
Idalécio Pacífico da Silva ${ }^{1}$, Fabiano Aurélio S. Oliveira ${ }^{2,3}$, Heloísa P. Pedroza ${ }^{3}$, \\ Ivana Cristina N. GadelHA ${ }^{1}$, Marília M. Melo ${ }^{3}$, Benito Soto-Blanco ${ }^{3}$ \\ ${ }^{1}$ Programa de Pós-graduação em Ciência Animal, Universidade Federal Rural do Semi-Árido (UFERSA), BR 110 Km \\ 47, Mossoró, RN 59625-900, Brazil \\ ${ }^{2}$ Laboratório Nacional Agropecuário (LANAGRO-MG), Ministério da Agricultura, Pecuária e Abastecimento (MAPA), \\ Av. Rômulo Joviano s/n, Caixa Postal 35/50, Pedro Leopoldo, MG 33600-000, Brazil \\ ${ }^{3}$ Departamento de Clínica e Cirurgia Veterinárias, Escola de Veterinária, Universidade Federal de Minas Gerais, Av. Pres. \\ Antônio Carlos 6627, Belo Horizonte, MG 31270-901, Brazil
}

Received 3 December 2014 - Revised 12 February 2015 - Accepted 4 March 2015

\begin{abstract}
The decline of honeybee (Apis mellifera L.) populations impacts global agricultural production and affects both food production and the economy. One of the probable causes for this decline is the indiscriminate use of pesticides. Here, we compare the levels of pesticide exposure among honeybees that are used to pollinate melon (Cucumis melo L.) crops, honeybees that forage in the forest, and stingless bees, Melipona subnitida, that forage in the forest. The level of pesticide exposure was determined by measuring residual pesticide levels of 152 compounds in the honey. Honey samples from the present study contained 19 different pesticides, 13 of which were present in honey from bees pollinating melon crops. The levels of some compounds were sufficiently high to promote toxic effects in the bees. Thus, crop pollination presents a toxicological risk to bees that may reduce their life span.
\end{abstract}

environmental contamination / insecticides / acaricides / herbicides / fungicides / nematicides / multiresidue analysis

\section{INTRODUCTION}

Flower pollination by animals is critical for agriculture; approximately $35 \%$ of crops are dependent on pollinators for sexual reproduction (Klein et al. 2007). The worldwide value of pollination in 2005 was estimated to be $€ 153$ billion (Gallai et al. 2009). Honeybees (Apis mellifera L.) are the most economically valuable pollinators for agriculture (Klein et al. 2007; Potts et al. 2010). However, recent declines in pollinator populations have affected global agricultural production and impacted

Corresponding author: B. Soto-Blanco, benito.blanco@pq.cnpq.br

Manuscript editor: Monique Gauthier both food production and the economy (Potts et al. 2010). One of the probable causes for the population declines of pollinators, including honeybees, is the indiscriminate use of pesticides (Klein et al. 2007; Potts et al. 2010; Nakasu et al. 2014).

Individual bees can be exposed directly through bodily contact with pesticides or indirectly by consuming pesticide residue in the nectar and pollen of flowers (Rortais et al. 2005). An entire colony may be exposed to pesticides through the collection and transportation of contaminated pollen by forager bees (Villa et al. 2000). Bees foraging on melon crops may also be exposed to pesticides via guttation fluid, a xylem sap exudate that is eliminated through leaf hydathodes (Thompson 2010; Hoffmann and Castle 2012). 
Pesticides can kill bees at sufficiently high doses (Rortais et al. 2005). However, pesticide doses that do not cause immediate death often have other deleterious effects and may interfere with the cognitive capacities and behavior of the bees. The potential negative consequences include impaired learning, orientation, and food collection abilities; affected bees may therefore have a reduced ability to collect food and navigate back to their hive (Rortais et al. 2005; Desneux et al. 2007; Godfray et al. 2014). Furthermore, some pesticides can reduce the resistance of bee to the intracellular parasite Nosema (Microsporidia) (Alaux et al. 2010; Pettis et al. 2012; Wu et al. 2012; Aufauvre et al. 2012; Di Prisco et al. 2013) and the immune response against viruses (Di Prisco et al. 2013).

The aim of the present study was to determine the levels of pesticide exposure among honeybees (A. mellifera) that are used to pollinate melon (Cucumis melo L.) crops and compare the pesticide exposure levels to those of honeybees that forage in the forest (caatinga, a xeric shrubland and thorn forest in northeastern Brazil) and stingless bees, Melipona subnitida Ducke (tribe Meliponini from the family Apidae), that forage in the forest (caatinga). The level of pesticide exposure was determined by measuring residual pesticide levels of 152 compounds in the honey. The stingless bee M. subnitida was in addition to honeybees that forage in the forest because the colonies kept in the study region did not collect pollen from muskmelons (Maia-Silva 2013).

\section{MATERIAL AND METHODS}

\subsection{Chemicals and materials}

All reagents were of analytical grade. Florisil, LCMS grade acetonitrile, and glacial acetic acid were supplied by Merck (Darmstadt, Germany). Methanol was obtained from Baker (Xalostoc, México). Analytical reagent grade anhydrous magnesium sulfate (purity $\geq 97 \%$ ) was purchased from Sigma-Aldrich, and anhydrous sodium acetate and ammonium acetate (purity $\geq 98 \%$ ) were purchased from Vetec (Rio de Janeiro, RJ, Brazil). Formic acid was purchased from Tedia (Ohio, USA). Ultrapure water was generated using a Millipore Milli-Q system (Milford, MA, USA). All of the standards used were of high purity grade $(>98.0 \%)$ and were purchased from Riedel-de Haën (Selze, Germany) or Sigma-Aldrich (Saint Louis, USA). Individual stock solutions were prepared at $1000 \mu \mathrm{g} \mathrm{L}^{-1}$ in either acetonitrile and stored at $-20 \pm 2{ }^{\circ} \mathrm{C}$. The working solutions were prepared as appropriate dilutions of the stock solutions.

\subsection{Samples}

Honey samples were collected from 23 colonies of honeybees (A. mellifera) used to pollinate melon crops, 20 colonies of honeybees (A. mellifera) that forage in the forest (caatinga), and 10 colonies of stingless bees (M. subnitida Ducke) that forage in the forest (caatinga). Samples were collected directly from two frames of each colony. All colonies were raised at the Mossoró ( $05^{\circ} 11^{\prime} 16^{\prime \prime} \mathrm{S}$ and $\left.37^{\circ} 20^{\prime} 38^{\prime \prime} \mathrm{W}\right)$ and Baraúna $\left(05^{\circ} 04^{\prime} 48^{\prime \prime} \mathrm{S}\right.$ and $\left.37^{\circ} 37^{\prime} 01^{\prime \prime} \mathrm{W}\right)$ municipalities, Rio Grande do Norte state, northeastern Brazil. All the colonies of bees that forage in the forest were at a minimal distance of $8 \mathrm{~km}$ from melon crops. No pesticide was used in the beehives to control parasites such as Varroa spp.

\subsection{Sample preparation}

Sample extraction and clean up (Rissato et al. 2006; Pittella 2009) were performed as follows. Honey samples $(10.0 \mathrm{~g})$ were transferred to polypropylene centrifuge tubes $(50 \mathrm{~mL})$ with $10.0 \mathrm{~mL}$ of deionized water. Then, $10.0 \mathrm{~mL}$ of ethyl acetate was added, and the tubes were shaken at $3000 \mathrm{rpm}$ for $1 \mathrm{~min}$. The tubes were then centrifuged at $4{ }^{\circ} \mathrm{C}, 2700 \times g$ for $9 \mathrm{~min}$. The supernatants were transferred to clean polypropylene centrifuge tubes $(50 \mathrm{~mL})$ and samples re-extracted three times with $5.0 \mathrm{~mL}$ of ethyl acetate. The combined ethyl acetate extracts $(25 \mathrm{~mL})$ were filtered using Florisil $(1 \mathrm{~g}$ packed in $6 \mathrm{~mL}$ cartridge) followed by magnesium sulfate (4.0 $\mathrm{g}$ in paper filter). Florisil and magnesium sulfate were used after heating overnight at $100{ }^{\circ} \mathrm{C}$. The extracts were dried at room temperature, resuspended in $1.0 \mathrm{~mL}$ of acetonitrile, transferred to vials, and analyzed using a UFLC-MS/MS system to identify different classes of pesticides (Table I). All identified pesticides were evaluated by UFLC-MS/MS using a multiresidue analysis technique. 
Table I. Pesticides surveyed in the honey samples.

\begin{tabular}{|c|c|c|c|}
\hline Pesticide & Use & Chemical group & Molecular formula \\
\hline 2,4-D (2,4-Dichlorophenoxyacetic acid) & $\mathrm{H}$ & Phenoxy acid & $\mathrm{C}_{8} \mathrm{H}_{6} \mathrm{Cl}_{2} \mathrm{O}_{3}$ \\
\hline 2,4-DB (4-(2,4-Dichlorophenoxy) butyric acid) & $\mathrm{H}$ & Phenoxy acid & $\mathrm{C}_{10} \mathrm{H}_{10} \mathrm{Cl}_{2} \mathrm{O}_{3}$ \\
\hline $2,4,5-\mathrm{T}(2,4,5$-Trichlorophenoxyacetic acid $)$ & $\mathrm{H}$ & Phenoxy acid & $\mathrm{C}_{8} \mathrm{H}_{5} \mathrm{Cl}_{3} \mathrm{O}_{3}$ \\
\hline 3-Hydroxycarbofuran & I & Carbamate & $\mathrm{C}_{12} \mathrm{H}_{15} \mathrm{NO}_{4}$ \\
\hline Acephate & I & Organophosphorus & $\mathrm{C}_{4} \mathrm{H}_{10} \mathrm{NO}_{3} \mathrm{PS}$ \\
\hline Acetamiprid & I & Neonicotinoid & $\mathrm{C}_{10} \mathrm{H}_{11} \mathrm{ClN}_{4}$ \\
\hline Aldicarb & I & Carbamate & $\mathrm{C}_{7} \mathrm{H}_{14} \mathrm{~N}_{2} \mathrm{O}_{2} \mathrm{~S}$ \\
\hline Aldicarb sulfone (metabolite) & I & Carbamate & $\mathrm{C}_{7} \mathrm{H}_{14} \mathrm{~N}_{2} \mathrm{O}_{4} \mathrm{~S}$ \\
\hline Aldicarbe sulfoxide (metabolite) & I & Carbamate & $\mathrm{C}_{7} \mathrm{H}_{14} \mathrm{~N}_{2} \mathrm{O}_{3} \mathrm{~S}$ \\
\hline Amitraz & $\mathrm{A} / \mathrm{I}$ & Amidine & $\mathrm{C}_{19} \mathrm{H}_{23} \mathrm{~N}_{3}$ \\
\hline Aramite & A & Sulfite ester & $\mathrm{C}_{15} \mathrm{H}_{23} \mathrm{ClO}_{4} \mathrm{~S}$ \\
\hline Azinphos-ethyl & $\mathrm{A} / \mathrm{I}$ & Organophosphorus & $\mathrm{C}_{12} \mathrm{H}_{16} \mathrm{~N}_{3} \mathrm{O}_{3} \mathrm{PS}_{2}$ \\
\hline Azinphos-methyl & I & Organophosphorus & $\mathrm{C}_{10} \mathrm{H}_{12} \mathrm{~N}_{3} \mathrm{O}_{3} \mathrm{PS}_{2}$ \\
\hline Azoxystrobin & $\mathrm{F}$ & Strobilurin & $\mathrm{C}_{22} \mathrm{H}_{17} \mathrm{~N}_{3} \mathrm{O}_{5}$ \\
\hline Barban & $\mathrm{H}$ & Carbamate & $\mathrm{C}_{11} \mathrm{H}_{9} \mathrm{Cl}_{2} \mathrm{NO}_{2}$ \\
\hline Benalaxyl & $\mathrm{H}$ & Acylalanine & $\mathrm{C}_{20} \mathrm{H}_{23} \mathrm{NO}_{3}$ \\
\hline Benfuracarb & $\mathrm{I} / \mathrm{N}$ & Carbamate & $\mathrm{C}_{20} \mathrm{H}_{30} \mathrm{~N}_{2} \mathrm{O}_{5} \mathrm{~S}$ \\
\hline Benomyl & $\mathrm{F}$ & Benzimidazole & $\mathrm{C}_{14} \mathrm{H}_{18} \mathrm{~N}_{4} \mathrm{O}_{3}$ \\
\hline Bentazon & $\mathrm{H}$ & Diazine & $\mathrm{C}_{10} \mathrm{H}_{12} \mathrm{~N}_{2} \mathrm{O}_{3} \mathrm{~S}$ \\
\hline BF 500-3 (metabolite pyraclostrobin) & $\mathrm{F}$ & Strobilurin & $\mathrm{C}_{18} \mathrm{H}_{16} \mathrm{Cl} \mathrm{N}{ }_{3} \mathrm{O}_{3}$ \\
\hline Bifenthrin & $\mathrm{A} / \mathrm{I}$ & Pyrethroid & $\mathrm{C}_{23} \mathrm{H}_{22} \mathrm{ClF}_{3} \mathrm{O}_{2}$ \\
\hline Boscalid & $\mathrm{F}$ & Anilide & $\mathrm{C}_{18} \mathrm{H}_{12} \mathrm{Cl}_{2} \mathrm{~N}_{2} \mathrm{O}$ \\
\hline Carbaryl & I & Carbamate & $\mathrm{C}_{12} \mathrm{H}_{11} \mathrm{NO}_{2}$ \\
\hline Carbendazim & $\mathrm{F}$ & Benzimidazole & $\mathrm{C}_{9} \mathrm{H}_{9} \mathrm{~N}_{3} \mathrm{O}_{2}$ \\
\hline Carbofuran & I & Carbamate & $\mathrm{C}_{12} \mathrm{H}_{15} \mathrm{NO}_{3}$ \\
\hline Carbosulfan & I & Carbamate & $\mathrm{C}_{20} \mathrm{H}_{32} \mathrm{~N}_{2} \mathrm{O}_{3} \mathrm{~S}$ \\
\hline Chlorbufam & $\mathrm{H}$ & Carbamate & $\mathrm{C}_{11} \mathrm{H}_{10} \mathrm{ClNO}_{2}$ \\
\hline Chlorfenvinphos & $\mathrm{A} / \mathrm{I}$ & Organophosphorus & $\mathrm{C}_{12} \mathrm{H}_{14} \mathrm{Cl}_{3} \mathrm{O}_{4} \mathrm{P}$ \\
\hline Chloroxuron & $\mathrm{H}$ & Urea & $\mathrm{C}_{15} \mathrm{H}_{15} \mathrm{ClN}_{2} \mathrm{O}_{2}$ \\
\hline Chlorpyrifos & $\mathrm{A} / \mathrm{I}$ & Organophosphorus & $\mathrm{C}_{9} \mathrm{H}_{11} \mathrm{Cl}_{3} \mathrm{NO}_{3} \mathrm{PS}$ \\
\hline Chlorpyrifos methyl & $\mathrm{A} / \mathrm{I}$ & Organophosphorus & $\mathrm{C}_{7} \mathrm{H}_{7} \mathrm{Cl}_{3} \mathrm{NO}_{3} \mathrm{PS}$ \\
\hline Cinidon-ethyl & $\mathrm{H}$ & Dicarboximide & $\mathrm{C}_{19} \mathrm{H}_{17} \mathrm{Cl}_{2} \mathrm{NO}_{4}$ \\
\hline Cyazofamid & $\mathrm{F}$ & Imidazole & $\mathrm{C}_{13} \mathrm{H}_{13} \mathrm{ClN}_{4} \mathrm{O}_{2} \mathrm{~S}$ \\
\hline Cymoxanil & $\mathrm{F}$ & Cyanoacetamide oximo & $\mathrm{C}_{7} \mathrm{H}_{10} \mathrm{~N}_{4} \mathrm{O}_{3}$ \\
\hline Cyproconazole & $\mathrm{F}$ & Triazole & $\mathrm{C}_{15} \mathrm{H}_{18} \mathrm{ClN}_{3} \mathrm{O}$ \\
\hline Cyprodinil & $\mathrm{F}$ & Anilinopyrimidine & $\mathrm{C}_{14} \mathrm{H}_{15} \mathrm{~N}_{3}$ \\
\hline Cyromazine & I & Triazine & $\mathrm{C}_{6} \mathrm{H}_{10} \mathrm{~N}_{6}$ \\
\hline Deltamethrin & $\mathrm{I} / \mathrm{F}$ & Pyrethroid & $\mathrm{C}_{22} \mathrm{H}_{19} \mathrm{Br}_{2} \mathrm{NO}_{3}$ \\
\hline Diallate & $\mathrm{A} / \mathrm{H}$ & Carbamate & $\mathrm{C}_{10} \mathrm{H}_{17} \mathrm{Cl}_{2} \mathrm{NOS}$ \\
\hline Diazinon & $\mathrm{A} / \mathrm{I} / \mathrm{N}$ & Organophosphorus & $\mathrm{C}_{12} \mathrm{H}_{21} \mathrm{~N}_{2} \mathrm{O}_{3} \mathrm{PS}$ \\
\hline Dichlorprop & $\mathrm{N}$ & Carboxylic acid & $\mathrm{C}_{9} \mathrm{H}_{8} \mathrm{Cl}_{2} \mathrm{O}_{3}$ \\
\hline Dichlorvos & I & Organophosphorus & $\mathrm{C}_{4} \mathrm{H}_{7} \mathrm{C}_{12} \mathrm{O}_{4} \mathrm{P}$ \\
\hline
\end{tabular}


Table I (continued)

\begin{tabular}{|c|c|c|c|}
\hline Pesticide & Use & Chemical group & Molecular formula \\
\hline Difenoconazole & $\mathrm{F}$ & Triazole & $\mathrm{C}_{19} \mathrm{H}_{17} \mathrm{Cl}_{2} \mathrm{~N}_{3} \mathrm{O}_{3}$ \\
\hline Diflubenzuron & I & Benzoylurea & $\mathrm{C}_{14} \mathrm{H}_{9} \mathrm{ClF}_{2} \mathrm{~N}_{2} \mathrm{O}_{2}$ \\
\hline Dimethoate & I & Organophosphorus & $\mathrm{C}_{5} \mathrm{H}_{12} \mathrm{NO}_{3} \mathrm{PS}_{2}$ \\
\hline Dinocap & $\mathrm{A} / \mathrm{F}$ & Dinitrophenol & $\mathrm{C}_{18} \mathrm{H}_{24} \mathrm{~N}_{2} \mathrm{O}_{6}$ \\
\hline Dinoseb & $\mathrm{H}$ & Dinitrophenol & $\mathrm{C}_{18} \mathrm{H}_{24} \mathrm{~N}_{2} \mathrm{O}_{6}$ \\
\hline Dinoterb & $\mathrm{H}$ & Dinitrophenol & $\mathrm{C}_{10} \mathrm{H}_{12} \mathrm{~N}_{2} \mathrm{O}_{5}$ \\
\hline Disulfoton & $\mathrm{A} / \mathrm{I}$ & Organophosphorus & $\mathrm{C}_{8} \mathrm{H}_{19} \mathrm{O}_{2} \mathrm{PS}_{3}$ \\
\hline Disulfoton sulfone (metabolite) & $\mathrm{A} / \mathrm{I}$ & Organophosphorus & $\mathrm{C}_{8} \mathrm{H}_{19} \mathrm{O}_{4} \mathrm{PS}_{3}$ \\
\hline Disulfoton sulfoxide (metabolite) & $\mathrm{A} / \mathrm{I}$ & Organophosphorus & $\mathrm{C}_{8} \mathrm{H}_{19} \mathrm{O}_{3} \mathrm{PS}_{3}$ \\
\hline Ethion & $\mathrm{A} / \mathrm{I}$ & Organophosphorus & $\mathrm{C}_{9} \mathrm{H}_{22} \mathrm{O}_{4} \mathrm{P}_{2} \mathrm{~S}_{4}$ \\
\hline Ethofumesate & $\mathrm{H}$ & Benzofuran & $\mathrm{C}_{13} \mathrm{H}_{18} \mathrm{O}_{5} \mathrm{~S}$ \\
\hline Ethoprophos & $\mathrm{I} / \mathrm{N}$ & Organophosphorus & $\mathrm{C}_{8} \mathrm{H}_{19} \mathrm{O}_{2} \mathrm{PS}_{2}$ \\
\hline Ethoxysulfuron & $\mathrm{H}$ & Sulfonylurea & $\mathrm{C}_{15} \mathrm{H}_{18} \mathrm{~N}_{4} \mathrm{O}_{7} \mathrm{~S}$ \\
\hline Etrimfos & $\mathrm{A} / \mathrm{I}$ & Organophosphorus & $\mathrm{C}_{10} \mathrm{H}_{17} \mathrm{~N}_{2} \mathrm{OPS}$ \\
\hline Fenamidone & $\mathrm{F}$ & Imidazolinone & $\mathrm{C}_{17} \mathrm{H}_{17} \mathrm{~N}_{3} \mathrm{OS}$ \\
\hline Fenamiphos & $\mathrm{N}$ & Organophosphorus & $\mathrm{C}_{13} \mathrm{H}_{22} \mathrm{NO}_{3} \mathrm{PS}$ \\
\hline Fenamiphos sulfone (metabolite) & $\mathrm{N}$ & Organophosphorus & $\mathrm{C}_{13} \mathrm{H}_{22} \mathrm{NO}_{5} \mathrm{PS}$ \\
\hline Fenamiphos sulfoxide (metabolite) & $\mathrm{N}$ & Organophosphorus & $\mathrm{C}_{13} \mathrm{H}_{22} \mathrm{NO}_{4} \mathrm{PS}$ \\
\hline Fenarimol & $\mathrm{F}$ & Pyrimidine & $\mathrm{C}_{17} \mathrm{H}_{12} \mathrm{Cl}_{2} \mathrm{~N}_{2} \mathrm{O}$ \\
\hline Fenhexamid & $\mathrm{F}$ & Hydroxyanilide & $\mathrm{C}_{14} \mathrm{H}_{17} \mathrm{Cl}_{2} \mathrm{NO}_{2}$ \\
\hline Fenpropimorph & $\mathrm{F}$ & Morpholine & $\mathrm{C}_{20} \mathrm{H}_{33} \mathrm{NO}$ \\
\hline Fenthion & I & Organophosphorus & $\mathrm{C}_{10} \mathrm{H}_{15} \mathrm{O}_{3} \mathrm{PS}_{2}$ \\
\hline Fenthion sulfoxide (metabolite) & I & Organophosphorus & $\mathrm{C}_{10} \mathrm{H}_{15} \mathrm{O}_{4} \mathrm{PS}_{2}$ \\
\hline Fipronil & I & Pyrazole & $\mathrm{C}_{12} \mathrm{H}_{4} \mathrm{Cl}_{2} \mathrm{~F}_{6} \mathrm{~N}_{4} \mathrm{OS}$ \\
\hline Fipronil sulfone (metabolite) & I & Pyrazole & $\mathrm{C}_{12} \mathrm{H}_{4} \mathrm{Cl}_{2} \mathrm{~F}_{6} \mathrm{~N}_{4} \mathrm{O}_{2} \mathrm{~S}$ \\
\hline Fluasifop-p-butyl & $\mathrm{H}$ & Aryloxyphenoxypropionate & $\mathrm{C}_{19} \mathrm{H}_{20} \mathrm{~F}_{3} \mathrm{NO}_{4}$ \\
\hline Fludioxonil & $\mathrm{F}$ & Phenylpyrrole & $\mathrm{C}_{12} \mathrm{H}_{6} \mathrm{~F}_{2} \mathrm{~N}_{2} \mathrm{O}_{2}$ \\
\hline Flumethrin & I & Pyrethroid & $\mathrm{C}_{28} \mathrm{H}_{22} \mathrm{Cl}_{2} \mathrm{FNO}_{3}$ \\
\hline Fluquinconazole & $\mathrm{F}$ & Triazole & $\mathrm{C}_{16} \mathrm{H}_{8} \mathrm{Cl}_{2} \mathrm{FN}_{5} \mathrm{O}$ \\
\hline Fluroxypyr & $\mathrm{H}$ & Auxin & $\mathrm{C}_{7} \mathrm{H}_{5} \mathrm{Cl}_{2} \mathrm{FN}_{2} \mathrm{O}_{3}$ \\
\hline Flutriafol & $\mathrm{F}$ & Triazole & $\mathrm{C}_{16} \mathrm{H}_{13} \mathrm{~F}_{2} \mathrm{~N}_{3} \mathrm{O}$ \\
\hline Foramsulfuron & $\mathrm{H}$ & Sulfonylurea & $\mathrm{C}_{17} \mathrm{H}_{20} \mathrm{~N}_{6} \mathrm{O}_{7} \mathrm{~S}$. \\
\hline Furathiocarb & I & Carbamate & $\mathrm{C}_{18} \mathrm{H}_{26} \mathrm{~N}_{2} \mathrm{O}_{5} \mathrm{~S}$ \\
\hline Hexaconazole & $\mathrm{F}$ & Triazole & $\mathrm{C}_{14} \mathrm{H}_{17} \mathrm{Cl}_{2} \mathrm{~N}_{3} \mathrm{O}$ \\
\hline Hexythiazox & A & Thiazolidinecarboxamide & $\mathrm{C}_{17} \mathrm{H}_{21} \mathrm{ClN}_{2} \mathrm{O}_{2} \mathrm{~S}$ \\
\hline Imazalil & $\mathrm{F}$ & Imidazole & $\mathrm{C}_{14} \mathrm{H}_{14} \mathrm{Cl}_{2} \mathrm{~N}_{2} \mathrm{O}$ \\
\hline Imidacloprid & I & Neonicotinoid & $\mathrm{C}_{9} \mathrm{H}_{10} \mathrm{ClN}_{5} \mathrm{O}_{2}$ \\
\hline Indoxacarb & I & Oxadiazine & $\mathrm{C}_{22} \mathrm{H}_{17} \mathrm{ClF}_{3} \mathrm{~N}_{3} \mathrm{O}_{7}$ \\
\hline Iprodione & $\mathrm{F}$ & Carboxamide & $\mathrm{C}_{13} \mathrm{H}_{13} \mathrm{Cl}_{2} \mathrm{~N}_{3} \mathrm{O}_{3}$ \\
\hline Iprovalicarb & $\mathrm{F}$ & Carbamate & $\mathrm{C}_{18} \mathrm{H}_{28} \mathrm{~N}_{2} \mathrm{O}_{3}$ \\
\hline Isoproturon & $\mathrm{H}$ & Urea & $\mathrm{C}_{12} \mathrm{H}_{18} \mathrm{~N}_{2} \mathrm{O}$ \\
\hline Isoxaflutole & $\mathrm{H}$ & Cyclopropylisoxazole & $\mathrm{C}_{15} \mathrm{H}_{12} \mathrm{~F}_{3} \mathrm{NO}_{4} \mathrm{~S}$ \\
\hline
\end{tabular}


Table I (continued)

\begin{tabular}{|c|c|c|c|}
\hline Pesticide & Use & Chemical group & Molecular formula \\
\hline Kresoxim-methyl & $\mathrm{F}$ & Strobilurin & $\mathrm{C}_{18} \mathrm{H}_{19} \mathrm{NO}_{4}$ \\
\hline Linuron & $\mathrm{H}$ & Urea & $\mathrm{C}_{9} \mathrm{H}_{10} \mathrm{Cl}_{2} \mathrm{~N}_{2} \mathrm{O}_{2}$ \\
\hline Malathion & I & Organophosphorus & $\mathrm{C}_{10} \mathrm{H}_{19} \mathrm{O}_{6} \mathrm{PS}_{2}$ \\
\hline Metalaxyl & $\mathrm{F}$ & Benzenoid & $\mathrm{C}_{15} \mathrm{H}_{21} \mathrm{NO}_{4}$ \\
\hline Metazachlor & $\mathrm{H}$ & Chloroacetanilide & $\mathrm{C}_{14} \mathrm{H}_{16} \mathrm{ClN}_{3} \mathrm{O}$ \\
\hline Metconazole & $\mathrm{F}$ & Triazole & $\mathrm{C}_{17} \mathrm{H}_{22} \mathrm{ClN}_{3} \mathrm{O}$ \\
\hline Methamidophos & I & Organophosphorus & $\mathrm{C}_{2} \mathrm{H}_{8} \mathrm{NO}_{2} \mathrm{PS}$ \\
\hline Methidathion & I & Organophosphorus & $\mathrm{C}_{6} \mathrm{H}_{11} \mathrm{~N}_{2} \mathrm{O}_{4} \mathrm{PS}_{3}$ \\
\hline Methidathion OA (metabolite) & I & Organophosphorus & $\mathrm{C}_{6} \mathrm{H}_{11} \mathrm{~N}_{2} \mathrm{O}_{4} \mathrm{PS}_{3}$ \\
\hline Methomyl & I & Carbamate & $\mathrm{C}_{5} \mathrm{H}_{10} \mathrm{~N}_{2} \mathrm{O}_{2} \mathrm{~S}$ \\
\hline Metsulfuton-methyl (metabolite) & $\mathrm{H}$ & Sulfonylurea & $\mathrm{C}_{14} \mathrm{H}_{15} \mathrm{~N}_{5} \mathrm{O}_{6} \mathrm{~S}$ \\
\hline Mevinphos & $\mathrm{A} / \mathrm{I}$ & Organophosphorus & $\mathrm{C}_{7} \mathrm{H}_{13} \mathrm{O}_{6} \mathrm{P}$ \\
\hline Monocrotophos & I & Organophosphorus & $\mathrm{C}_{7} \mathrm{H}_{14} \mathrm{NO}_{5} \mathrm{P}$ \\
\hline Monolinuron & $\mathrm{H}$ & Urea & $\mathrm{C}_{9} \mathrm{H}_{11} \mathrm{ClN}_{2} \mathrm{O}_{2}$ \\
\hline Myclobutanil & $\mathrm{F}$ & Triazole & $\mathrm{C}_{15} \mathrm{H}_{17} \mathrm{ClN}_{4}$ \\
\hline Omethoate & I & Organophosphorus & $\mathrm{C}_{5} \mathrm{H}_{12} \mathrm{NO}_{4} \mathrm{PS}$ \\
\hline Oxamyl & I & Carbamate & $\mathrm{C}_{7} \mathrm{H}_{13} \mathrm{~N}_{3} \mathrm{O}_{3} \mathrm{~S}$ \\
\hline Oxasulfuron & $\mathrm{H}$ & Sulfonylurea & $\mathrm{C}_{17} \mathrm{H}_{18} \mathrm{~N}_{4} \mathrm{O}_{6} \mathrm{~S}$ \\
\hline Oxyfluorfen & $\mathrm{H}$ & Nitrophrnyl ether & $\mathrm{C}_{15} \mathrm{H}_{11} \mathrm{ClF}_{3} \mathrm{NO}_{4}$ \\
\hline Paraoxon (metabolite) & I & Organophosphorus & $\mathrm{C}_{10} \mathrm{H}_{14} \mathrm{NO}_{6} \mathrm{P}$ \\
\hline Parathion-ethyl & $\mathrm{A} / \mathrm{I}$ & Organophosphorus & $\mathrm{C}_{10} \mathrm{H}_{14} \mathrm{NO}_{5} \mathrm{PS}$ \\
\hline Penconazole & $\mathrm{F}$ & Triazole & $\mathrm{C}_{13} \mathrm{H}_{15} \mathrm{Cl}_{2} \mathrm{~N}_{3}$ \\
\hline Pencycuron & $\mathrm{F}$ & Phenilurea & $\mathrm{C}_{19} \mathrm{H}_{21} \mathrm{ClN}_{2} \mathrm{O}$ \\
\hline Pendimethalin & $\mathrm{H}$ & Dinitroaniline & $\mathrm{C}_{13} \mathrm{H}_{19} \mathrm{~N}_{3} \mathrm{O}_{4}$ \\
\hline Phenthoato & $\mathrm{A} / \mathrm{I}$ & Organophosphorus & $\mathrm{C}_{12} \mathrm{H}_{17} \mathrm{O}_{4} \mathrm{PS}_{2}$ \\
\hline Phorate & $\mathrm{A} / \mathrm{I} / \mathrm{N}$ & Organophosphorus & $\mathrm{C}_{7} \mathrm{H}_{17} \mathrm{O}_{2} \mathrm{PS}_{3}$ \\
\hline Phorate sulfoxide (metabolite) & $\mathrm{A} / \mathrm{I} / \mathrm{N}$ & Organophosphorus & $\mathrm{C}_{7} \mathrm{H}_{17} \mathrm{O}_{3} \mathrm{PS}_{3}$ \\
\hline Phosalone & $\mathrm{A} / \mathrm{I}$ & Organophosphorus & $\mathrm{C}_{12} \mathrm{H}_{15} \mathrm{ClNO}_{4} \mathrm{PS}_{2}$ \\
\hline Phosmet & $\mathrm{A} / \mathrm{I}$ & Organophosphorus & $\mathrm{C}_{11} \mathrm{H}_{12} \mathrm{NO}_{4} \mathrm{PS}_{2}$ \\
\hline Picolinafen & $\mathrm{H}$ & Anilide & $\mathrm{C}_{19} \mathrm{H}_{12} \mathrm{~F}_{4} \mathrm{~N}_{2} \mathrm{O}_{2}$ \\
\hline Pirimicarb & I & Carbamate & $\mathrm{C}_{11} \mathrm{H}_{18} \mathrm{~N}_{4} \mathrm{O}_{2}$ \\
\hline Pirimiphos ethyl & $\mathrm{A} / \mathrm{I}$ & Organophosphorus & $\mathrm{C}_{13} \mathrm{H}_{24} \mathrm{~N}_{3} \mathrm{O}_{3} \mathrm{PS}$ \\
\hline Pirimiphos methyl & $\mathrm{A} / \mathrm{I}$ & Organophosphorus & $\mathrm{C}_{11} \mathrm{H}_{20} \mathrm{~N}_{3} \mathrm{O}_{3} \mathrm{PS}$ \\
\hline Prochloraz & $\mathrm{F}$ & Imidazole & $\mathrm{C}_{15} \mathrm{H}_{16} \mathrm{Cl}_{3} \mathrm{~N}_{3} \mathrm{O}_{2}$ \\
\hline Profenofos & I & Organophosphorus & $\mathrm{C}_{11} \mathrm{H}_{15} \mathrm{BrClO}_{3} \mathrm{PS}$ \\
\hline Propargite & $\mathrm{H}$ & Sulfite ester & $\mathrm{C}_{19} \mathrm{H}_{26} \mathrm{O}_{4} \mathrm{~S}$ \\
\hline Propham & $\mathrm{H}$ & Carbamate & $\mathrm{C}_{10} \mathrm{H}_{13} \mathrm{NO}_{2}$ \\
\hline Propiconazole & $\mathrm{F}$ & Triazole & $\mathrm{C}_{15} \mathrm{H}_{17} \mathrm{Cl}_{2} \mathrm{~N}_{3} \mathrm{O}_{2}$ \\
\hline Propoxur & I & Carbamate & $\mathrm{C}_{11} \mathrm{H}_{15} \mathrm{NO}_{3}$ \\
\hline Propyzamide & $\mathrm{H}$ & Benzimidazole & $\mathrm{C}_{12} \mathrm{H}_{11} \mathrm{Cl}_{2} \mathrm{NO}$ \\
\hline Prosulfuron & $\mathrm{H}$ & Sulfonylurea & $\mathrm{C}_{15} \mathrm{H}_{16} \mathrm{~F}_{3} \mathrm{~N}_{5} \mathrm{O}_{4} \mathrm{~S}$ \\
\hline Pymetrozine & I & Triazine & $\mathrm{C}_{10} \mathrm{H}_{11} \mathrm{~N}_{5} \mathrm{O}$ \\
\hline
\end{tabular}


Table I (continued)

\begin{tabular}{|c|c|c|c|}
\hline Pesticide & Use & Chemical group & Molecular formula \\
\hline Pyraclostrobin & $\mathrm{F}$ & Strobilurin & $\mathrm{C}_{19} \mathrm{H}_{18} \mathrm{CIN}_{3} \mathrm{O}_{4}$ \\
\hline Pyrazophos & $\mathrm{F}$ & Organophosphorus & $\mathrm{C}_{14} \mathrm{H}_{20} \mathrm{~N}_{3} \mathrm{O}_{5} \mathrm{PS}$ \\
\hline Pyridaben & I & Unclassified & $\mathrm{C}_{19} \mathrm{H}_{25} \mathrm{CIN}_{2} \mathrm{OS}$ \\
\hline Pyridate & $\mathrm{H}$ & Pyridazine & $\mathrm{C}_{19} \mathrm{H}_{23} \mathrm{ClN}_{2} \mathrm{O}_{2} \mathrm{~S}$ \\
\hline Pyrimethanil & $\mathrm{F}$ & Anilinopyrimidine & $\mathrm{C}_{12} \mathrm{H}_{13} \mathrm{~N}_{3}$ \\
\hline Quinalphos & $\mathrm{A} / \mathrm{I}$ & Organophosphorus & $\mathrm{C}_{12} \mathrm{H}_{15} \mathrm{~N}_{2} \mathrm{O}_{3} \mathrm{PS}$ \\
\hline Spiroxamine & $\mathrm{F}$ & Morpholine & $\mathrm{C}_{18} \mathrm{H}_{35} \mathrm{NO}_{2}$ \\
\hline Sulfotep & I & Organophosphorus & $\mathrm{C}_{8} \mathrm{H}_{20} \mathrm{O}_{5} \mathrm{P}_{2} \mathrm{~S}_{2}$ \\
\hline Tebuconazole & $\mathrm{F}$ & Triazole & $\mathrm{C}_{16} \mathrm{H}_{22} \mathrm{ClN}_{3} \mathrm{O}$ \\
\hline Tebufenozide & I & Dimethylbenzohydrazide & $\mathrm{C}_{22} \mathrm{H}_{28} \mathrm{~N}_{2} \mathrm{O}_{2}$ \\
\hline TEPP (tetraethyl pyrophosphate) & $\mathrm{A} / \mathrm{I}$ & Organophosphorus & $\mathrm{C}_{8} \mathrm{H}_{20} \mathrm{O}_{7} \mathrm{P}_{2}$ \\
\hline Thiacloprid & I & Neonicotinoid & $\mathrm{C}_{10} \mathrm{H}_{9} \mathrm{CIN}_{4} \mathrm{~S}$ \\
\hline Thiamethoxam & I & Neonicotinoid & $\mathrm{C}_{8} \mathrm{H}_{10} \mathrm{ClN}_{5} \mathrm{O}_{3} \mathrm{~S}$ \\
\hline Thifensulfuron methyl & $\mathrm{H}$ & Sulfonylurea & $\mathrm{C}_{12} \mathrm{H}_{13} \mathrm{~N}_{5} \mathrm{O}_{6} \mathrm{~S}_{2}$ \\
\hline Thiodicarb & I & Carbamate & $\mathrm{C}_{10} \mathrm{H}_{18} \mathrm{~N}_{4} \mathrm{O}_{4} \mathrm{~S}_{3}$ \\
\hline Thiophanate-methyl & $\mathrm{F}$ & Benzimidazole & $\mathrm{C}_{12} \mathrm{H}_{14} \mathrm{~N}_{4} \mathrm{O}_{4} \mathrm{~S}_{2}$ \\
\hline Tiabendazole & $\mathrm{F}$ & Benzimidazole & $\mathrm{C}_{10} \mathrm{H}_{7} \mathrm{~N}_{3} \mathrm{~S}$ \\
\hline Tolylfluanid & $\mathrm{F}$ & Phenylsulfamide & $\mathrm{C}_{10} \mathrm{H}_{13} \mathrm{Cl}_{2} \mathrm{FN}_{2} \mathrm{O}_{2} \mathrm{~S}_{2}$ \\
\hline Triadimefon & $\mathrm{F}$ & Triazole & $\mathrm{C}_{14} \mathrm{H}_{16} \mathrm{ClN}_{3} \mathrm{O}_{2}$ \\
\hline Triadimenol & $\mathrm{F}$ & Triazole & $\mathrm{C}_{14} \mathrm{H}_{18} \mathrm{ClN}_{3} \mathrm{O}_{2}$ \\
\hline Triasulfuron & $\mathrm{H}$ & Sulfonylurea & $\mathrm{C}_{14} \mathrm{H}_{16} \mathrm{ClN}_{5} \mathrm{O}_{5} \mathrm{~S}$ \\
\hline Triazophos & $\mathrm{I} / \mathrm{A} / \mathrm{N}$ & Organophosphorus & $\mathrm{C}_{12} \mathrm{H}_{16} \mathrm{~N}_{3} \mathrm{O}_{3} \mathrm{PS}$ \\
\hline Trichlorfon & I & Organophosphorus & $\mathrm{C}_{4} \mathrm{H}_{8} \mathrm{Cl}_{3} \mathrm{O}_{4} \mathrm{P}$ \\
\hline Tridemorph & $\mathrm{F}$ & Morpholine & $\mathrm{C}_{19} \mathrm{H}_{39} \mathrm{NO}$ \\
\hline Trifloxystrobin & $\mathrm{F}$ & Strobilurin & $\mathrm{C}_{20} \mathrm{H}_{19} \mathrm{~F}_{3} \mathrm{~N}_{2} \mathrm{O}_{4}$ \\
\hline Triforine & $\mathrm{F} / \mathrm{I}$ & Piperazine & $\mathrm{C}_{10} \mathrm{H}_{14} \mathrm{Cl}_{6} \mathrm{~N}_{4} \mathrm{O}_{2}$ \\
\hline
\end{tabular}

$I$ insecticide, $A$ acaricide, $H$ herbicide, $F$ fungicide, $N$ nematicide

\subsection{Chromatographic conditions}

Chromatographic analyses were performed using a UFLC system (Shimadzu LC20 ADXR) equipped with a binary pump (Shimadzu LC20ADXR), an auto sampler (Shimadzu SIL20ACXR), and a column oven (Shimadzu CTO20AC). The separations were achieved using a Shim-pack XR-ODSII column $(2.0 \times 100 \mathrm{~mm}$, 2.2- $\mu \mathrm{m}$ particle size; Shimadzu). Chromatographic separation was carried out with a mobile phase consisting of ammonium acetate $\left(10 \mathrm{mmol} \mathrm{L}^{-1}\right)$ acidified with $0.01 \%$ formic acid (phase A) and methanol (phase B) at a flow rate of $0.5 \mathrm{~mL} \mathrm{~min}{ }^{-1}$. The gradient elution program was as follows: A (50\%)-B (50\%) (6 min), A (20\%)-B (80\%) (5 min), A (10\%)-B (90\%) (4 min), and A $(50 \%)-\mathrm{B}(50 \%)(3.0 \mathrm{~min})$. The total chromatographic run time was $13 \mathrm{~min}$. Injection volume was $5 \mu \mathrm{L}$, and the column temperature was set at $60^{\circ} \mathrm{C}$.

\subsection{Mass spectrometric conditions}

Mass spectrometry analysis was carried out using a 5500 Triple Quad mass spectrometer (Applied Biosystems, MDS SCIEX, Ontario, Canada). The instrument was operated using an electrospray ionization source (ESI) in both positive and negative ion modes. Instrument settings, data acquisition, and data processing were controlled by the Analyst software program (Version 1.5.1, Applied Biosystems). Source parameters were optimized as follows: ion spray voltage, $5.5 \mathrm{kV}$ for 
ESI (+) and $4.5 \mathrm{kV}$ for ESI (-); curtain gas, 20 psi; collision gas, 8 psi; nebulizer gas and auxiliary gas, $30 \mathrm{psi}$; ion source temperature, $500{ }^{\circ} \mathrm{C}$.

Calibration curves were performed using acetonitrile as a solvent to standardize the results of recovery and simplify the experiment. The calibration levels were as follows: 5.0, 7.5, 10.0, 25.0, 50.0, 75.0, and $100 \mu \mathrm{g} \mathrm{L}{ }^{-1}$ (where this sequence was randomly injected; $n=6$ ). All solutions were prepared independently. For simultaneous quantification and identification purposes, two multiple reaction monitoring (MRM) transitions for each analyte were used to avoid false negatives at trace pesticide levels. The peaks were evenly distributed along the chromatographic window and were resolved symmetrically. The analytical curve was also prepared in extract matrix free of the studied analytes to compensate the matrix effect. The data were analyzed using the Analyst program (Version 1.5.1, Applied Biosystems). The model for the regression curve for each compound was selected by applying a homoscedasticity test. The fit quality and significance of the regression model employed were evaluated using the lack-of-fit test. The limit of detection (LOD) and the limit of quantification (LOQ) for all tested pesticides were determined to be 5.0 and $10.0 \mu \mathrm{g} \mathrm{kg}^{-1}$, respectively. Spiked experiments at levels of 10.0 and $50.0 \mu \mathrm{g} \mathrm{kg}^{-1}$ showed that recoveries ranged from 70 to $120 \%$ for all compounds.

\subsection{Statistical analyses}

The obtained data were statistically analyzed using R (version 3.0.3) (R Development Core Team 2008) with the "agricolae" package. The positive sample frequencies for pesticide residues were compared using Fisher's exact test. The mean, median, the standard deviation (SD), the standard error of the mean (SEM), and the range of pesticide concentrations were calculated using all analyzed samples, even those with undetectable concentrations. For the compounds that were not detected (below the LOD), the concentration used for statistical analysis was half of the LOD $\left(2.5 \mu \mathrm{g} \mathrm{kg}^{-1}\right)$. For the compounds that were detected (above the LOD) but were not quantified (below the LOQ), the concentration used for statistical analysis was the mean of the LOD and the LOQ (7.5 $\mu \mathrm{g} \mathrm{kg}^{-1}$ ) (Lambert et al. 2013). Data normality was evaluated using Shapiro-Wilk's test, and the homogeneity of variances was evaluated using the Bartlett's test. The concentrations were compared using the Kruskal-Wallis test followed by the Student-Newman-Keuls test. The level of statistical significance was set to $P<0.05$.

\section{RESULTS}

A total of 19 pesticides were found in the tested honey samples. Thirteen compounds were found in the honey from honeybees used to pollinate melon crops (Table II), six compounds were found in the honey from honeybees that forage in the forest (Table III), and four compounds were found in the honey from stingless bees that forage in the forest (Table IV).

The number of unique pesticides detected in the honey samples was higher $(P<0.0001)$ for honeybees that pollinate melon crops than for honeybees and stingless bees that forage in the forest. The number of positive samples for each pesticide is presented in Table V. The honey from honeybees pollinating muskmelon presented higher frequencies $(P<0.05)$ of samples positive for acetamiprid, carbaryl, chlorpyrifos, furathiocarb, imidacloprid, paraoxon, parathionethyl, sulfotep, and thiamethoxam. The honey from honeybees foraging in the forest presented higher frequencies $(P<0.05)$ of samples positive for azoxystrobin, bifenthrin, myclobutanil, and thiophanate-methyl. Dimethoate displayed higher frequencies $(P<0.05)$ in honey from honeybees and stingless bees foraging in the forest than from honeybees pollinating melon crops.

The concentrations of pesticides detected in the honey samples are shown in Table VI. Honey from the honeybees used to pollinate melon crops had the highest $(P<0.05)$ concentrations of carbaryl, chlorpyrifos, imidacloprid, paraoxon, parathion-ethyl, sulfotep, and thiamethoxam. The concentration of thiophanate-methyl was highest $(P<0.05)$ in the honey from honeybees that forage in the forest. The concentration of dimethoate in honey from stingless bees was higher than in the honey from honeybees that pollinate melon crops but was not significantly different from that in the honey from honeybees that forage in the forest. 
Table II. Pesticides (in $\mu \mathrm{g} \mathrm{kg}^{-1}$ ) detected in the honey from honeybees (A. mellifera ) used to pollinate muskmelon.

\begin{tabular}{lllcrc}
\hline Pesticide & Positive samples & Minimum & Maximum & Median & SD \\
\hline Acetamiprid & $6(26.1 \%)$ & $<$ LOD & 32.3 & 2.50 & 8.23 \\
Aldicarb sulfoxide & $1(4.3 \%)$ & $<$ LOD & 32.8 & 2.50 & 6.32 \\
Carbaryl & $23(100 \%)$ & 41.9 & 418.9 & 114.1 & 87.5 \\
Carbofuran & $3(13.0 \%)$ & $<$ LOD & $<$ LOQ & 2.50 & 1.72 \\
Chlorpyrifos & $23(100 \%)$ & 14.3 & 32.4 & 20.4 & 6.01 \\
Ethion & $1(4.3 \%)$ & $<$ LOD & 13.3 & 2.50 & 2.24 \\
Furathiocarb & $19(82.6 \%)$ & $<$ LOD & $<$ LOQ & 7.50 & 1.94 \\
Imidacloprid & $7(30.4 \%)$ & $<$ LOD & 106.0 & 2.50 & 38.8 \\
Paraoxon & $21(91.3 \%)$ & $<$ LOD & 59.6 & 7.50 & 17.0 \\
Parathion-ethyl & $23(100 \%)$ & 118.4 & 2912.1 & 645.0 & 914.3 \\
Phosalone & $1(4.3 \%)$ & $<$ LOD & $<$ LOQ & 2.50 & 1.04 \\
Sulfotep & $5(21.7 \%)$ & $<$ LOD & 11.1 & 2.50 & 2.95 \\
Thiamethoxam & $8(34.8 \%)$ & $<$ LOD & 19.1 & 2.50 & 5.44 \\
\hline
\end{tabular}

\section{DISCUSSION AND CONCLUSION}

Of the 19 compounds found in honey samples in our study, seven (chlorpyrifos, ethion, paraoxon, parathion, phosalone, and sulfotep) belong to the organophosphorus chemical group, and four (aldicarb sulfoxyde, carbaryl, carbofuran, and furathiocarb) are carbamates. All of these compounds were found in the honey from honeybees that pollinate melon crops. Both organophosphorus and carbamate insecticides are known to inhibit cholinesterase, the enzyme that hydrolyses the neurotransmitter acetylcholine (Sultatos 2005). Honeybees are known to be very sensitive to even a single exposure of carbamate (Akca et al. 2009; Hardstone and Scott 2010) and organophosphorus insecticides (Abrol and Andotra
2003; Hardstone and Scott 2010), which result in high mortality within $24 \mathrm{~h}$ after exposure. In fact, the LD50 for topical exposure to dimethoate, chlorpyrifos, and carbaryl in honeybees was determined to be $22.4,35.4$, and $42.8 \mathrm{ng}$ per bee (Abrol and Andotra 2003), which corresponds to approximately 862,1362 , and $1646 \mu \mathrm{g} \mathrm{kg}^{-1}$ in bee food, respectively. These values are higher than the concentrations found in the honey samples during our study. However, sub-lethal doses may elicit behavioral changes in bees. For example, the frequency of visits to the feeder in honeybees treated topically with $50 \mathrm{ng}$ parathion per bee was increased compared to control bees, but at $10 \mathrm{ng}$ per bee, the frequency primarily decreased and then increased (Guez et al. 2005). Thus, residual levels of some organophosphorus and

Table III. Pesticides (in $\mu \mathrm{g} \mathrm{kg}^{-1}$ ) detected in the honey from honeybees (A. mellifera) that forage in the forest (caatinga).

\begin{tabular}{lllccc}
\hline Pesticide & Positive samples & Minimum & Maximum & Median & SD \\
\hline Azoxystrobin & $6(30 \%)$ & $<$ LOD & 60.0 & 2.50 & 13.5 \\
Bifenthrin & $5(25 \%)$ & $<$ LOD & $<$ LOQ & 2.50 & 2.22 \\
Dimethoate & $4(20 \%)$ & $<$ LOD & 11.0 & 2.50 & 2.76 \\
Iprodione & $2(10 \%)$ & $<$ LOD & $<$ LOQ & 2.50 & 1.54 \\
Myclobutanil & $4(20 \%)$ & $<$ LOD & 27.0 & 2.50 & 6.42 \\
Thiophanate-methyl & $8(40 \%)$ & $<$ LOD & 33.0 & 2.50 & 11.4 \\
\hline
\end{tabular}


Table IV. Pesticides (in $\mu \mathrm{g} \mathrm{kg}^{-1}$ ) detected in the honey from stingless bees (M. subnitida) that forage in the forest (caatinga).

\begin{tabular}{llllll}
\hline Pesticide & Positive samples & Minimum & Maximum & Median & SD \\
\hline Bifenthrin & $1(10 \%)$ & $<$ LOD & $<$ LOQ & 2.50 & 1.58 \\
Dimethoate & $3(30 \%)$ & $<$ LOD & 14.0 & 2.50 & 3.89 \\
Iprodione & $1(10 \%)$ & $<$ LOD & 10.0 & 2.50 & 2.37 \\
Myclobutanil & $2(20 \%)$ & $<$ LOD & $<$ LOQ & 2.50 & 2.11 \\
\hline
\end{tabular}

carbamate insecticides might impact the feeding behavior of bees used to pollinate muskmelon.

The neonicotinoid insecticides imidacloprid, acetamiprid, and thiamethoxam were found in the honey of bees that pollinate melon crops. Application of neonicotinoid insecticides to plants is known to result in residual pesticide concentrations in the nectar and pollen of flowers, even when it is used to treat only the seeds (Schmuck et al. 2001; Bonmatin et al. 2003; Rortais et al. 2005). Furthermore, the guttation drops of muskmelon treated with imidacloprid may contain high concentrations of this compound (up to $37 \mu \mathrm{g} / \mathrm{mL}$ ) (Hoffmann and Castle 2012). Neonicotinoid insecticides act as agonists of the nicotinic

Table V. Number of samples with detected pesticides in honey from honeybees (A. mellifera) pollinating melon crops or foraging in the forest (caatinga) and from stingless bees (M. subnitida) foraging in the forest.

\begin{tabular}{lllll}
\hline Pesticide & Melon $(n=23)$ & Forest $(n=20)$ & M. subnitida $(n=10)$ & $P^{\mathrm{a}}$ \\
\hline Acetamiprid & $6(26.1 \%)$ & 0 & 0 & 0.0148 \\
Aldicarb sulfoxide & $1(4.3 \%)$ & 0 & 0 & n.s. \\
Azoxystrobin & 0 & $6(30 \%)$ & 0 & 0.0022 \\
Bifenthrin & 0 & $5(25 \%)$ & $1(10 \%)$ & 0.0243 \\
Carbaryl & $23(100 \%)$ & 0 & 0 & $<0.0001$ \\
Carbofuran & $3(13.0 \%)$ & 0 & 0 & n.s. \\
Chlorpyrifos & $23(100 \%)$ & 0 & 0 & $<0.0001$ \\
Dimethoate & 0 & $4(20 \%)$ & $3(30 \%)$ & 0.0139 \\
Ethion & $1(4.3 \%)$ & 0 & 0 & n.s. \\
Furathiocarb & $19(82.6 \%)$ & 0 & 0 & $<0.0001$ \\
Imidacloprid & $7(30.4 \%)$ & 0 & 0 & 0.0052 \\
Iprodione & 0 & $2(10 \%)$ & $1(10 \%)$ & n.s. \\
Myclobutanil & 0 & $4(20 \%)$ & $2(20 \%)$ & 0.0463 \\
Paraoxon & $21(91.3 \%)$ & 0 & 0 & $<0.0001$ \\
Parathion-ethyl & $23(100 \%)$ & 0 & 0 & $<0.0001$ \\
Phosalone & $1(4.3 \%)$ & 0 & 0 & n.s. \\
Sulfotep & $5(21.7 \%)$ & 0 & 0 & 0.0389 \\
Thiamethoxam & $8(34.8 \%)$ & 0 & 0 & 0.0017 \\
Thiophanate-methyl & 0 & $8(40 \%)$ & 0 & 0.0004 \\
\hline
\end{tabular}

n.s. $P>0.05$

${ }^{\mathrm{a}}$ Fisher test 
Table VI. Concentrations of detected pesticides (in $\mu \mathrm{g} \mathrm{kg}^{-1}$ ) in honey from honeybees (A. mellifera) pollinating melon crops or foraging in the forest (caating $a$ ) and from stingless bees (M. subnitida) foraging in the forest. Data are shown as the mean $\pm \mathrm{SEM}$ of positive and negative results.

\begin{tabular}{lclcc}
\hline Pesticide & Melon $(n=23)$ & Forest $(n=20)$ & M. subnitida $(n=10)$ & $P^{\mathrm{a}}$ \\
\hline Acetamiprid & $5.87 \pm 1.72 \mathrm{a}$ & $2.50 \pm 0 \mathrm{a}$ & $2.50 \pm 0 \mathrm{a}$ & 0.0134 \\
Aldicarb sulfoxide & $3.82 \pm 1.32$ & $2.50 \pm 0$ & $2.50 \pm 0$ & n.s. \\
Azoxystrobin & $2.50 \pm 0 \mathrm{a}$ & $8.32 \pm 3.03 \mathrm{a}$ & $2.50 \pm 0 \mathrm{a}$ & 0.0043 \\
Bifenthrin & $2.50 \pm 0 \mathrm{a}$ & $3.75 \pm 0.50 \mathrm{a}$ & $3.00 \pm 0.50 \mathrm{a}$ & 0.0377 \\
Carbaryl & $139.1 \pm 18.3 \mathrm{a}$ & $2.50 \pm 0 \mathrm{~b}$ & $2.50 \pm 0 \mathrm{~b}$ & $<0.0001$ \\
Carbofuran & $3.15 \pm 0.36$ & $2.50 \pm 0$ & $2.50 \pm 0$ & n.s. \\
Chlorpyrifos & $21.8 \pm 1.25 \mathrm{a}$ & $2.50 \pm 0 \mathrm{~b}$ & $2.50 \pm 0 \mathrm{~b}$ & $<0.0001$ \\
Dimethoate & $2.50 \pm 0 \mathrm{a}$ & $3.80 \pm 0.62 \mathrm{a}, \mathrm{b}$ & $4.65 \pm 1.23 \mathrm{~b}$ & 0.0368 \\
Ethion & $2.97 \pm 0.47$ & $2.50 \pm 0$ & $2.50 \pm 0$ & n.s. \\
Furathiocarb & $6.63 \pm 0.40 \mathrm{a}$ & $2.50 \pm 0 \mathrm{~b}$ & $2.50 \pm 0 \mathrm{~b}$ & $<0.0001$ \\
Imidacloprid & $26.8 \pm 8.10 \mathrm{a}$ & $2.50 \pm 0 \mathrm{~b}$ & $2.50 \pm 0 \mathrm{~b}$ & 0.0059 \\
Iprodione & $2.50 \pm 0$ & $3.00 \pm .34$ & $3.25 \pm 0.75$ & n.s. \\
Myclobutanil & $2.50 \pm 0$ & $5.12 \pm 1.44$ & $3.50 \pm 0.67$ & n.s. \\
Paraoxon & $18.5 \pm 3.55 \mathrm{a}$ & $2.50 \pm 0 \mathrm{~b}$ & $2.50 \pm 0 \mathrm{~b}$ & $<0.0001$ \\
Parathion-ethyl & $880.0 \pm 190.7 \mathrm{a}$ & $2.50 \pm 0 \mathrm{~b}$ & $2.50 \pm 0 \mathrm{~b}$ & $<0.0001$ \\
Phosalone & $2.72 \pm 0.22$ & $2.50 \pm 0$ & $2.50 \pm 0$ & n.s. \\
Sulfotep & $3.98 \pm 0.62 \mathrm{a}$ & $2.50 \pm 0 \mathrm{~b}$ & $2.50 \pm 0 \mathrm{~b}$ & 0.0295 \\
Thiamethoxam & $6.04 \pm 1.3 \mathrm{a}$ & $2.50 \pm 0 \mathrm{~b}$ & $2.50 \pm 0 \mathrm{~b}$ & 0.0025 \\
Thiophanate-methyl & $2.50 \pm 0 \mathrm{a}$ & $10.2 \pm 2.56 \mathrm{~b}$ & $2.50 \pm 0 \mathrm{a}$ & 0.0005 \\
\hline
\end{tabular}

Different letters in the same row indicate a significant difference between values (Student-Newman-Keuls test)

n.s. $P>0.05$

${ }^{a}$ Kruskal-Wallis test

acetylcholine receptor ( $\mathrm{nAChR})$ in a similar way as nicotine, but with much higher potency and selectivity to the receptors of insects than of mammals (Tomizawa and Casida 2008). The LD50 of acute oral exposure to imidacloprid in honeybees was estimated to be 3.7 to $40.9 \mathrm{ng}$ per bee, or 0.14 to $1.57 \mathrm{mg} \mathrm{kg}^{-1}$ in food (Schmuck et al. 2001), and the LD50 of acute oral exposure to thiamethoxam in honeybees was estimated to be $5.0 \mathrm{ng}$ per bee (Godfray et al. 2014). However, bees are much more sensitive to chronic exposure to imidacloprid: reduced survival rates were observed in honeybees that ingested a cumulative dose of $0.01 \mathrm{ng}$ per bee for 8 days (Suchail et al. 2001).

Imidacloprid can reduce the resistance of bees to pathogens (Alaux et al. 2010; Pettis et al. 2012). Doses as low as $5 \mu \mathrm{g} \mathrm{kg}$ given to bees via sucrose syrup increased the number of spores per bee of the gut pathogen Nosema (Pettis et al. 2012). In addition to its immunosuppressive effects, imidacloprid also promotes behavioral disturbances. Honeybees that ingested $50 \mu \mathrm{g} \mathrm{kg}^{-1}$ imidacloprid in their food did not travel as far and spent more time near food sources (Teeters et al. 2012). In addition, this compound reduced the olfactory memory of honeybees at doses of $12 \mathrm{ng}$ per bee (Decourtye et al. 2004; Decourtye et al. 2005). Furthermore, Apis cerana that were fed nectar containing $34 \mu \mathrm{g} \mathrm{kg}^{-1}$ imidacloprid showed reduced hornet predator avoidance, and those fed either 17 or $34 \mu \mathrm{g} \mathrm{kg}^{-1}$ collected a lower volume of 
nectar (Tan et al. 2014). As honey from bees used to pollinate muskmelon presented up to $106.0 \mu \mathrm{g} \mathrm{kg}^{-1}$ of imidacloprid, these bees may display foraging behavior changes and immune suppression. In addition, residual imidacloprid may persist in the contaminated honey for several months, further increasing the risk to these bees (Rortais et al. 2005).

In our study, the pyrethroid bifenthrin was found at low levels $(<\mathrm{LOQ})$ in the honey from honeybees and stingless bees that forage in the forest. Pyrethroids, including bifenthrin, are known to decrease the neuronal excitability of neurons in the honeybee brain by decreasing the sodium currents (Zhou et al. 2011). Honeybees are very sensitive to bifenthrin, and exposure may be lethal (Qualls et al. 2010). Furthermore, bifenthrin was reported to negatively affect bees by reducing fecundity and growth and by prolonging the immature phases of life (Dai et al. 2010). The concentration of bifenthrin in honey was very low (less than $5 \mu \mathrm{g} \mathrm{kg}^{-1}$ ); however, its presence in the honey is indicative of environmental contamination after inadequate use, such as incorrect pesticide concentration and spraying and indiscriminate disposition of empty containers.

Four of the detected compounds in the honey from bees that forage in the forest act as fungicides: azoxystrobin (strobilurin), iprodione (carboxamide), myclobutanil (triazole), and thiophanate-methyl (benzimidazole). Iprodione was found to be non-toxic to honeybees and Osmia lignaria (a solitary bee) after direct contact and oral exposure (Ladurner et al. 2005). Fungicides may affect bees indirectly by reducing the populations of fungi that are beneficial to bees. These fungi convert the stored pollen by fermentation into food for larvae, known as bee bread (Yoder et al. 2013).

In summary, honey samples from the present study showed 19 different pesticides, 13 of which were present in the honey from bees that pollinate melon crops. The levels of several compounds were high enough to promote adverse effects in the bees. Thus, crop pollination presents a toxicological risk to bees that may reduce their life span.

\section{ACKNOWLEDGMENTS}

This research was supported by a grant from the CNPq (project no. 305761/2013-7), and language editing was provided by the Pró-Reitoria de Pesquisa of the Universidade Federal de Minas Gerais (Edital PRP-UFMG 01/2014).

OPEN ACCESS This article is distributed under the terms of the Creative Commons Attribution License which permits any use, distribution, and reproduction in any medium, provided the original author(s) and the source are credited.

Exposition à des pesticides par des abeilles (Apis mellifera ) pollinisant des cultures de melon

contamination environnementale / insecticide / acaricide / herbicice / fongicide / nematicide / analyse multi résiduelle

Pesitizidexposition von Honigbienen (Apis mellifera) bei der Bestäubung von Melonen

Kontamination durch die Umwelt / Insektizide Akarizide / Herbizide / Fungizide / Nematizide Multi -Rückstandsuntersuchu

\section{REFERENCES}

Abrol, D.P., Andotra, R.S. (2003) Relative toxicity of some insecticides to Apis mellifera L. J. Asia Pac. Entomol. 6(2), 235-237

Akca, I., Tuncer, C., Guler, A., Saruhan, I. (2009) Residual toxicity of 8 different insecticides on honey bee (Apis mellifera, Hymenoptera: Apidae). J. Anim. Vet. Adv. 8(3), 436-440

Alaux, C., Brunet, J.L., Dussaubat, C., Mondet, F., Tchamitchan, S., Cousin, M., Brillard, J., Baldy, A., Belzunces, L.P., Le Conte, Y. (2010) Interactions between Nosema microspores and a neonicotinoid weaken honeybees (Apis mellifera). Environ. Microbiol. $12(3), 774-782$

Aufauvre, J., Biron, D.G., Vidau, C., Fontbonne, R., Roudel, M., Diogon, M., Viguès, B., Belzunces, L.P., Delbac, F., Blot, N. (2012) Parasite-insecticide interactions: a case study of Nosema ceranae and fipronil synergy on honeybee. Sci. Rep. 2, 326

Bonmatin, J.M., Moineau, I., Charvet, R., Fleche, C., Colin, M.E., Bengsch, E.R. (2003) A LC/APCI-MS/ MS method for analysis of imidacloprid in soils, in plants, and in pollens. Anal. Chem. 75(9), 2027-2033 
Dai, P.L., Wang, Q., Sun, J.H., Liu, F., Wang, X., Wu, Y.-Y., Zhou, T. (2010) Effects of sublethal concentrations of bifenthrin and deltamethrin on fecundity, growth, and development of the honeybee Apis mellifera ligustica . Environ. Toxicol. Chem. 29(3), 644-649

Decourtye, A., Armengaud, C., Renou, M., Devillers, J., Cluzeau, S., Gauthier, M., Pham-Delègue, M.H. (2004) Imidacloprid impairs memory and brain metabolism in the honeybee (Apis mellifera L.). Pestic. Biochem. Physiol 78(2), 83-92

Decourtye, A., Devillers, J., Genecque, E., Le Menach, K., Budzinski, H., Cluzeau, S., Pham-Delègue, M.H. (2005) Comparative sublethal toxicity of nine pesticides on olfactory learning performances of the honeybee Apis mellifera . Arch. Environ. Contam. Toxicol. 48 (2), 242-250

Desneux, N., Decourtye, A., Delpuech, J.M. (2007) The sublethal effects of pesticides on beneficial arthropods. Annu. Rev. Entomol. 52, 81-106

Development Core Team, R. (2008) R: A language and environment for statistical computing. R Foundation for Statistical Computing, Vienna, Austria

Di Prisco, G., Cavaliere, V., Annoscia, D., Varricchio, P., Caprio, E., Nazzi, F., Gargiulo, G., Francesco Pennacchio, F. (2013) Neonicotinoid clothianidin adversely affects insect immunity and promotes replication of a viral pathogen in honey bees. Proc. Natl. Acad. Sci. 110 (46), 18466-18471

Gallai, N., Salles, J.-M., Settele, J., Vaissière, B.E. (2009) Economic valuation of the vulnerability of world agriculture confronted with pollinator decline. Ecol. Econ. 68 (3), 810-821

Godfray, H.C.J., Blacquière, T., Field, L.M., Hails, R.S., Petrokofsky, G., Potts, S.G., Raine, N.E., Vanbergen, A.J., McLean, A.R. (2014) A restatement of the natural science evidence base concerning neonicotinoid insecticides and insect pollinators. Proc. Biol. Sci. d281(1786), 20140558

Guez, D., Zhang, S.-W., Srinivasan, M. (2005) Methyl parathion modifies foraging behaviour in honeybees (Apis mellifera). Ecotoxicology 14 (4), 431-437

Hardstone, M.C., Scott, J.G. (2010) Is Apis mellifera more sensitive to insecticides than other insects? Pest Manag. Sci. 66 (11), 1171-1180

Hoffmann, E.J., Castle, S.J. (2012) Imidacloprid in melon guttation fluid: a potential mode of exposure for pest and beneficial organisms. J. Econ. Entomol. 105(1), $67-71$

Klein, A.-M., Vaissière, B.E., Cane, J.H., SteffanDewenter, I., Cunningham, S.A., Kremen, C., Tscharntke, T. (2007) Importance of pollinators in changing landscapes for world crops. Proc. Biol. Sci. 274 (1608), 303-313

Ladurner, E., Bosch, J., Kemp, W.P., Maini, S. (2005) Assessing delayed and acute toxicity of five formulated fungicides to Osmia lignaria Say and Apis mellifera. Apidologie 36 (3), 449-460

Lambert, O., Piroux, M., Puyo, S., Thorin, C., L'Hostis, M., Wiest, L., Buleté, A., Delbac, F., Pouliquen, H. (2013)
Widespread occurrence of chemical residues in beehive matrices from apiaries located in different landscapes of western France. PLoS One 8(6), e67007

Maia-Silva C. (2013) Adaptações comportamentais de Melipona subnitida (Apidae, Meliponini) às condições ambientais do semiárido brasileiro. $\mathrm{PhD}$ Thesis, Universidade de São Paulo

Nakasu, E.Y.T., Williamson, S.M., Edwards, M.G., Fitches, E.C., Gatehouse, J.A., Wright, G.A., Gatehouse, A.M. (2014) Novel biopesticide based on a spider venom peptide shows no adverse effects on honeybees. Proc. Biol. Sci. 281 (1787), 20140619

Pettis, J., van Engelsdorp, D., Johnson, J., Dively, G. (2012) Pesticide exposure in honey bees results in increased levels of the gut pathogen Nosema. Naturwissenschaften 99 (2), 153-158

Pittella, C.M. (2009) Determinação de resíduos de agrotóxicos em mel de abelhas (Apis sp) por cromatografia gasosa acoplada à espectrometria de massas. Dissertation, Universidade Federal de Minas Gerais

Potts, S.G., Biesmeijer, J.C., Kremen, C., Neumann, P., Schweiger, O., Kunin, W.E. (2010) Global pollinator declines: trends, impacts and drivers. Trends Ecol. Evol. 25 (6), 345-353

Qualls, W.A., Xue, R.-D., Zhong, H. (2010) Impact of bifenthrin on honeybees and Culex quinquefasciatus . J. Am. Mosq. Control Assoc. 26 (2), 223-225

Rissato, S.R., Galhiane, M.S., Knoll, F.R.N., Andrade, R.M.B., Almeida, M.V. (2006) Método multirresíduo para monitoramento de contaminação ambiental de pesticidas na região de Bauru (SP) usando mel como bio-indicador. Química Nova 29 (5), 950-955

Rortais, A., Arnold, G., Halm, M.-P., Touffet-Briens, F. (2005) Modes of honeybees exposure to systemic insecticides: estimated amounts of contaminated pollen and nectar consumed by different categories of bees. Apidologie 36 (1), 71-83

Schmuck, R., Schöning, R., Stork, A., Schramel, O. (2001) Risk posed to honeybees (Apis mellifera L, Hymenoptera) by an imidacloprid seed dressing of sunflowers. Pest. Manag. Sci. 57 (3), 225-238

Suchail, S., Guez, D., Belzunces, L.P. (2001) Discrepancy between acute and chronic toxicity induced by imidacloprid and its metabolites in Apis mellifera. Environ. Toxicol. Chem. 20 (11), 2482-2486

Sultatos, L.G. (2005) Interactions of organophosphorus and carbamate compounds with cholinesterases. In: Gupta, R.C. (ed.) Toxicology of organophosphate \& carbamate compounds, pp. 209-218. Academic, Burlington

Tan, K., Chen, W., Dong, S., Liu, X., Wang, Y., Nieh, J.C. (2014) Imidacloprid alters foraging and decreases bee avoidance of predators. PLoS One 9 (7), e102725

Teeters, B.S., Johnson, R.M., Ellis, M.D., Siegfried, B.D. (2012) Using video-tracking to assess sublethal effects of pesticides on honey bees (Apis mellifera L.). Environ. Toxicol. Chem 31 (6), 1349-1354

Thompson, H.M. (2010) Risk assessment for honey bees and pesticides-recent developments and "new issues”. Pest. Manag. Sci. 66(11), 1157-1162 
Tomizawa, M., Casida, J.E. (2008) Molecular recognition of neonicotinoid insecticides: the determinants of life or death. Acc. Chem. Res. $42(2)$, 260-269

Villa, S., Vighi, M., Finizio, A., Bolchi Serini, G. (2000) Risk assessment for honeybees from pesticide-exposed pollen. Ecotoxicology 9(4), 287-297

Wu, J.Y., Smart, M.D., Anelli, C.M., Sheppard, W.S. (2012) Honey bees (Apis mellifera) reared in brood combs containing high levels of pesticide residues exhibit increased susceptibility to Nosema
(Microsporidia) infection. J. Invertebr. Pathol. $109(3), 326-329$

Yoder, J.A., Jajack, A.J., Rosselot, A.E., Smith, T.J., Yerke, M.C., Sammataro, D. (2013) Fungicide contamination reduces beneficial fungi in bee bread based on an areawide field study in honey bee, Apis mellifera, colonies. J. Toxicol. Environ. Health A 76(10), 587-600

Zhou, T., Zhou, W., Wang, Q., Dai, P.-L., Liu, F., Zhang, Y.L., Sun, J.-H. (2011) Effects of pyrethroids on neuronal excitability of adult honeybees Apis mellifera. Pestic. Biochem. Physiol. 100 (1), 35-40 